\title{
Sufficient Stochastic Maximum Principle for Discounted Control Problem
}

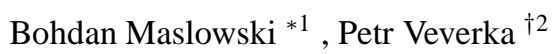 \\ ${ }^{1}$ Faculty of Mathematics and Physics, Charles University in Prague, Czech Republic, \\ maslow@karlin.mff.cuni.cz \\ ${ }^{2}$ Faculty of Nuclear Sciences and Physical Engineering, Czech Technical University in \\ Prague, Czech Republic, petr.veverka@fjfi.cvut.cz
}

\begin{abstract}
In this article, the sufficient Pontryagin's maximum principle for infinite horizon discounted stochastic control problem is established. The sufficiency is ensured by an additional assumption of concavity of the Hamiltonian function. Throughout the paper, it is assumed that the control domain $U$ is a convex set and the control may enter the diffusion term of the state equation. The general results are applied to the controlled stochastic logistic equation of population dynamics.
\end{abstract}

Key Words: Stochastic maximum principle, discounted control problem, FBSDE, infinite horizon, stochastic logistic equation.

AMS Subject Classification: 60H10, 93E20.

\section{INTRODUCTION}

In the present paper, the discounted stochastic optimal control problem is dealt with. This kind of problem is very popular and plentifully used in many domains, especially in stochastic finance since it leads to maximizing the average discounted agent's utility. The method of solving the problem used here is the maximum principle which, in deterministic setting, was formulated in 1950s by the group of L. S. Pontryagin. For diffusions, the maximum principle has been studied by many researchers. The earliest versions of a maximum principle for such process were suggested by Kushner [14] and Bismut [5]. Further progress on the subject was subsequently made by Bensoussan [4], Peng [22], Cadenillas and Haussmann [7] and others. Originally, the main technical tool used when considering maximum principle was the calculus of variations which was not easy to apply to real examples and was not convenient for numerical simulations. The turning point which led to its further intensive study was the paper by Pardoux and Peng [21] where the general (nonlinear) problem of Backward Stochastic Differential Equation (BSDE in short) was formulated and the existence and uniqueness theorems were given. BSDE theory provides an elegant and easy-to-handle tool to describe the adjoint processes and to formulate the maximum principle by means of the Hamiltonian function. Also, numerical methods for this kind of processes are intensively studied.

These results were extended in numerous papers. For example, in case of diffusions with jumps, a necessary maximum principle on the finite time horizon was formulated by Tang and $\mathrm{Li}$ [25] whereas sufficient optimality conditions on finite time horizon were specified by Øksendal, Sulem and Framstad [17]. Maximum principle on infinite time horizon was studied in Haadem, Proske and Øksendal [12]. Interested reader can also find a large amount of papers on maximum principle for a variety of cases: Singular control (Bahlali and Mezerdi [3], Dufour and Miller [9], Øksendal and Sulem [16]), Impulse control (Chikodza [8], Wu and Zhang [28]), Controlled SPDE's (Al-Hussein [2], Fuhrman, Hu and Tessitore [11]), Delayed controlled systems (Agram, Haadem, Øksendal and Proske [1], Øksendal, Sulem and Zhang [18]), Near-optimal control (Zhou [29]) and many others.

Applying the Hamiltonian formalism to stochastic control problems, the class of Forward-Backward Stochastic Differential Equations (FBSDE in short) naturally arises in form of a partially-coupled system of the forward equation for the controlled diffusion and the adjoint backward equation for „generalized Lagrange multipliers". FBSDE with infinite (or random) time horizon are still subject to intensive study. The question which is quite delicate is the behaviour of the solution processes at infinity. There are several papers (e.g. Pardoux [19], Peng and Shi [23], Yin [26], Wu [27]) answering this question under different sets of assumptions both on the coefficients of the FBSDE

\footnotetext{
*The author was supported in part by the GACR grant No. P201/10/0752.

${ }^{\dagger}$ The author was supported by the Czech CTU grant SGS12/197/OHK4/3T/14, MSMT grant INGO II INFRA LG12020 and GACR GRANT No. P201/10/0752.
} 
and on the terminal condition. Some of these papers naturally require that the terminal condition of the backward equation at infinity (in certain sense) is given in advance with suitable properties that consequently determine the space for the solution processes. Unfortunately, this is not directly applicable to stochastic control problems on infinite time horizon since the terminal condition is usually not known. Therefore, a different approach has to be introduced, namely we specify the solution space of random functions which determines asymptotical behaviour of the processes, suitable for our purpose. This approach appears already in Peng and Shi [23] where however the conditions on coefficients, are rather restrictive - global Lipschitz property in all variables is assumed (which, in fact, excludes polynomial coefficients of higher order than two) and some special kind of so called weak monotonicity (also known as one-sided Lipschitz property). In this case, the solution process is vanishing at infinity and therefore, the terminal condition is zero a.s. In the paper by Wu [27], a different monotonicity condition is assumed to obtain a solution process with non zero (in general) yet still a.s. constant terminal condition. The most general result is due to Yin [26] who weakens the assumptions to obtain the solution in some exponentially-weighted $\mathbf{L}^{2}$ space for some suitable discount factor. Nevertheless, in the latter paper, existence of the solution to the backward part of the system employs the result by Pardoux [19] based on the knowledge of the terminal condition.

The novelty of present paper is twofold: First of all, the applicability of the maximum principle to infinite time horizon control problems has been studied under a natural set of conditions that are verifiable and may cover nonlinear problems with non-Lipschitz coefficients. Secondly, these general results have been used to solve the corresponding control problem for controlled stochastic logistic equation (which indeed involves non-Lipschitz terms), which is an important model of population dynamics. In this case also the solvability of the closed-loop FBSDE has been established.

The paper is organized as follows: The discounted control problem is formulated in the second section. In the third section the existence and uniqueness theorem of the solution to FBSDE on infinite time horizon is provided. Fourth section contains the main result of the paper - the sufficient infinite time maximum principle for the discounted problem. In the fifth section, some standard examples are solved by using results of the fourth section to show the applicability. The last section which contains our main example is devoted to the above mentioned controlled stochastic logistic equation.

\section{FORMULATION OF THE PROBLEM}

2.1. Preliminaries. We are given a basic probability space $(\Omega, \mathcal{F}, \mathbf{P})$ with $\mathbb{R}^{d}$-valued standard Wiener process $W=\left(W_{t}\right)_{t \geq 0}$. Let $\left(\mathcal{F}_{t}^{W}\right)_{t \geq 0}$ be the canonical filtration of $W$, i.e. $\mathcal{F}_{t}^{W}=\sigma\left(W_{s} ; s \leq\right.$ $t$ ), and $\left(\mathcal{F}_{t}\right)_{t \geq 0}$ be its $\mathbf{P}-$ null sets augmentation. We denote $\mathcal{F}_{\infty}=\bigvee_{t \geq 0} \mathcal{F}_{t} \subset \mathcal{F}$. Further, to simplify the notation, we write just 'a.s.' instead of ' $\mathbf{P}$-a.s.'. We denote $|\cdot|$ and $\|\cdot\|$ the Euclidean norms in $\mathbb{R}^{n}$ and $\mathbb{R}^{n \times d}$ respectively. Further, $\langle\cdot, \cdot\rangle$ stands for standard scalar product in $\mathbb{R}^{n}$ and $\operatorname{Tr}(\cdot)$ denotes the trace of a square matrix.

Now, for $\beta \in \mathbb{R}$ and any Banach space $\mathcal{X}$ with norm $\|\cdot\|_{\mathcal{X}}$, we introduce the space of random processes

$$
\begin{aligned}
\mathbf{L}_{\mathcal{F}}^{2, \beta}\left(\mathbb{R}_{+} ; \mathcal{X}\right):=\left\{v: \mathbb{R}_{+} \times \Omega \rightarrow \mathcal{X}: v \text { is }\left(\mathcal{F}_{t}\right)_{t \geq 0}-\right. & \text { progressive with } \\
& \left.\mathbf{E} \int_{0}^{+\infty} e^{\beta t}\left\|v_{t}\right\|_{\mathcal{X}}^{2} d t<+\infty\right\} .
\end{aligned}
$$

We denote $\mathbf{L}_{\mathcal{F}}^{2,0}\left(\mathbb{R}_{+} ; \mathcal{X}\right)$ just as $\mathbf{L}_{\mathcal{F}}^{2}\left(\mathbb{R}_{+} ; \mathcal{X}\right)$ and

$$
\begin{aligned}
& \mathbf{L}_{\mathcal{F}, l o c}^{2, \beta}\left(\mathbb{R}_{+} ; \mathcal{X}\right):=\left\{v: \mathbb{R}_{+} \times \Omega \rightarrow \mathcal{X}: v \text { is }\left(\mathcal{F}_{t}\right)_{t \geq 0}-\right.\text { progressive with } \\
& \left.\qquad \mathbf{E} \int_{0}^{T} e^{\beta t}\left\|v_{t}\right\|_{\mathcal{X}}^{2} d t<+\infty, \forall T>0\right\} .
\end{aligned}
$$

2.2. Discounted control problem. The controlled state process $\left(X_{t}\right)_{t \geq 0}$ is a strong solution to the following controlled SDE in $\mathbb{R}^{n}$ 


$$
\begin{aligned}
d X_{t} & =b\left(X_{t}, u_{t}\right) d t+\sigma\left(X_{t}, u_{t}\right) d W_{t}, \quad \forall t \geq 0, \\
X_{0} & =x
\end{aligned}
$$

where $U$ is a convex subset of $\mathbb{R}^{k}$, the mappings $b: \mathbb{R}^{n} \times U \rightarrow \mathbb{R}^{n}$ and $\sigma: \mathbb{R}^{n} \times U \rightarrow \mathbb{R}^{n \times d}$ satisfy conditions specified in the next section.

Denote $\mathcal{U}_{a d}$ the set of all admissible controls as

$$
\mathcal{U}_{a d}=\left\{u: \mathbb{R}_{+} \times \Omega \rightarrow U: u \in \mathbf{L}_{\mathcal{F}, l o c}^{2}(0,+\infty ; U)\right\}
$$

Any process $u(\cdot) \in \mathcal{U}_{a d}$ is called an admissible control. The cost functional takes the form

$$
J(u(\cdot))=\mathbf{E} \int_{0}^{+\infty} e^{-\beta t} f\left(X_{t}, u_{t}\right) d t
$$

where $f: \mathbb{R}^{n} \times U \rightarrow \mathbb{R}$ is the penalization (or appreciation) function such that $J(u(\cdot))$ converges for every admissible control and $\beta>0$ is the discount factor.

Further, define the value function $v$ by

$$
v=\sup _{u(\cdot) \in \mathcal{U}_{a d}} J(u(\cdot)) .
$$

The goal is to find such a strategy $u^{*}(\cdot) \in \mathcal{U}_{a d}$ so that the supremum in (2.6) is attained in $u^{*}(\cdot)$, i.e. $v=J\left(u^{*}(\cdot)\right)$. For the discounted control problem 2.3, 2.4, 2.5) and 2.6) we use the abbreviation DCP.

Define a generalized Hamiltonian function $\mathcal{H}$ associated to control problem 2.3 - 2.6 by $\mathcal{H}$ : $\mathbb{R}^{n} \times U \times \mathbb{R}^{n} \times \mathbb{R}^{n \times d} \rightarrow \mathbb{R}$ as

$$
\mathcal{H}(x, u, y, z)=\langle b(x, u), y\rangle+\operatorname{Tr}\left(\sigma(x, u)^{\prime} z\right)+f(x, u)-\beta\langle x, y\rangle .
$$

The Hamiltonian is an analogue to the Lagrange function in the theory of constrained optimization since the variables $y$ and $z$ can be viewed as 'generalized Lagrange multipliers' and the functions $b$ and $\sigma$ as the constraints for the dynamics of the space process $X_{t}$. The additional term $-\beta\langle x, y\rangle$ comes up from the Lyapunov function of the FBSDE system, see Peng, Shi [23].

We note that neither the extremal point of $\mathcal{H}$ w.r.t. $u$ nor the concavity/convexity w.r.t. $(x, u)$ do not depend on the last term $-\beta\langle x, y\rangle$.

Suppose further that $\mathcal{H}$ is differentiable in $x$ (with the gradient denoted as $\nabla_{x} \mathcal{H}$ ) and we consider the following BSDE

$$
-d Y_{t}=\nabla_{x} \mathcal{H}\left(X_{t}, u_{t}, Y_{t}, Z_{t}\right) d t-Z_{t} d W_{t}, \quad \forall t \geq 0,
$$

which, in application to the DCP, reads

$$
-d Y_{t}=\left(\nabla_{x} b\left(X_{t}, u_{t}\right) Y_{t}+D_{x} \sigma\left(X_{t}, u_{t}\right) \cdot Z_{t}+\nabla_{x} f\left(X_{t}, u_{t}\right)-\beta Y_{t}\right) d t-Z_{t} d W_{t}, \quad \forall t \geq 0 .
$$

We use the notation $D_{x} \sigma(x, u) \cdot z=\sum_{i=1}^{d} \nabla_{x} \sigma^{i}(x, u) z^{i} \in \mathbb{R}^{n}$, for $z \in \mathbb{R}^{n \times d}$, where $\sigma^{i}$ denotes the $i$-th column of the matrix $\sigma$.

\section{SOLUTION TO CONTROLLED DECOUPLED FBSDE ON INFINITE TIME HORIZON}

In this section, we study the following controlled FBSDE associated to the DCP

$$
\begin{aligned}
d X_{t} & =b\left(X_{t}, u_{t}\right) d t+\sigma\left(X_{t}, u_{t}\right) d W_{t}, \forall t \geq 0 \\
X_{0} & =x \in \mathbb{R}^{n}, \\
-d Y_{t} & =\left(\nabla_{x} b\left(X_{t}, u_{t}\right) Y_{t}+D_{x} \sigma\left(X_{t}, u_{t}\right) \cdot Z_{t}+\nabla_{x} f\left(X_{t}, u_{t}\right)-\beta Y_{t}\right) d t-Z_{t} d W_{t}, \forall t \geq 0 .
\end{aligned}
$$

Since the system 3.1 is partially-coupled, i.e. the forward part does not depend on the solution of the backward equation, we can solve the forward part separately on $\mathbb{R}_{+}$obtaining the process $X$. In the next step, plugging the solution $X$ into the backward part, we find the solution processes $(Y, Z)$. We start with results on infinite time horizon SDE which is given by 


$$
\begin{aligned}
d X_{t} & =b\left(X_{t}, u_{t}\right) d t+\sigma\left(X_{t}, u_{t}\right) d W_{t}, \quad \forall t \geq 0 \\
X_{0} & =x
\end{aligned}
$$

where $U$ is a convex bounded subset of $\mathbb{R}^{k}$ and $x \in \mathbb{R}^{n}$ is a deterministic initial condition. We further assume that the mappings

$$
\begin{aligned}
& b: \mathbb{R}^{n} \times U \rightarrow \mathbb{R}^{n}, \\
& \sigma: \mathbb{R}^{n} \times U \rightarrow \mathbb{R}^{n \times d},
\end{aligned}
$$

satisfy the following conditions.

(H1): $b(\cdot, \cdot)$ and $\sigma(\cdot, \cdot)$ are continuous on $\mathbb{R}^{n} \times \bar{U}$.

(H2): There exists $\mu_{1} \in \mathbb{R}$ such that

$$
\left\langle x_{1}-x_{2}, b\left(x_{1}, u\right)-b\left(x_{2}, u\right)\right\rangle \leq \mu_{1}\left|x_{1}-x_{2}\right|^{2},
$$

for any $\left(x_{1}, x_{2}, u\right) \in \mathbb{R}^{n} \times \mathbb{R}^{n} \times U$.

(H3): (Lipschitz property) There exists a constant $L>0$ such that

$$
\left\|\sigma\left(x_{1}, u\right)-\sigma\left(x_{2}, u\right)\right\| \leq L\left|x_{1}-x_{2}\right|
$$

for any $\left(x_{1}, x_{2}, u\right) \in \mathbb{R}^{n} \times \mathbb{R}^{n} \times U$.

The following choice of the solution space will allow to apply Theorem 1 bellow to sufficient maximum principle. As we will see, the suitable solution space ensuring proper asymptotics is the space $\mathbf{L}_{\mathcal{F}}^{2,-\beta}\left(\mathbb{R}_{+} ; \mathbb{R}^{n}\right)$ for some $\beta>0$. The following theorem holds.

Theorem 1. [Existence and uniqueness] Let assumptions (H1)-(H3) hold. Then for each control $u(\cdot) \in \mathcal{U}_{\text {ad }}$ and every initial condition $x \in \mathbb{R}^{n}, \operatorname{SDE}\left[3.2\right.$ admits a unique solution $X=X^{x, u(\cdot)} \in$ $\mathbf{L}_{\mathcal{F}}^{2,-\beta}\left(\mathbb{R}_{+} ; \mathbb{R}^{n}\right)$ where $\beta-2 \mu_{1}-2 L^{2}>0$.

Proof: Uniqueness follows from Lemma1 1 below. The proof of existence is similar to Theorem 2.2. in Friedman [10] and it will be only sketched. First note that by virtue of (H1)-(H3) we have that for all $\varepsilon>0$ there are constants $K_{\varepsilon}, \bar{K}(\bar{K}$ independent of $\varepsilon$ ) such that

$$
\begin{aligned}
\langle x, b(x, u)\rangle & \leq\left(\mu_{1}+\varepsilon\right)|x|^{2}+K_{\varepsilon}, \\
\|\sigma(x, u)\| & \leq L|x|+\bar{K},
\end{aligned}
$$

for all $x>0, u \in U$. Now for all $n \in \mathbb{N}$ define

$$
\tau_{n}=\inf \left\{t>0:\left|X_{t}^{n}\right| \geq n\right\},
$$

where $\left(X_{t}^{n}\right)$ is a local solution obtained for trimmed coefficients on a ball of radius $n$ and for time $t \in\left[0, \tau_{n}\right]$. Due to (H1)-(H3) and 3.5 one obtains

$$
\begin{aligned}
\sup _{t \geq 0} \mathbf{E} & {\left[e^{-\beta\left(t \wedge \tau_{n}\right)}\left|X_{t \wedge \tau_{n}}^{n}\right|^{2}\right]+\left(\beta-2\left(\mu_{1}+\varepsilon\right)-2 L^{2}\right) \mathbf{E} \int_{0}^{\tau_{n}} e^{-\beta\left(s \wedge \tau_{n}\right)}\left|X_{s \wedge \tau_{n}}^{n}\right|^{2} d s } \\
& \leq\left|x_{0}\right|^{2}+\frac{2}{\beta}\left(K_{\varepsilon}+\bar{K}^{2}\right)<+\infty .
\end{aligned}
$$

A similar estimate can be easily obtained with the supremum inside the expectation. By using the standard consistency argument and Lyapunov techniques with Lyapunov function $V(x)=1+|x|^{2}$ we conclude that there is a limit process $\left(X_{t}\right)_{t \geq 0}$ in $\mathbf{L}_{\mathcal{F}}^{2,-\beta}\left(\mathbb{R}_{+} ; \mathbb{R}^{n}\right)$ with $\beta-2 \mu_{1}-2 L^{2}>0$.

Lemma 1. [A priori estimate for SDE] Let assumptions $(H 1)-(H 3)$ hold and $u(\cdot) \in \mathcal{U}_{\text {ad }}$ be arbitrary but fixed. Let $X^{1}=X^{u(\cdot), 1}$ and $X^{2}=X^{u(\cdot), 2}$ be two solutions to (3.2) with initial values $x_{1}$ and $x_{2}$ respectively, belonging to $\mathbf{L}_{\mathcal{F}}^{2,-\beta}\left(\mathbb{R}_{+} ; \mathbb{R}^{n}\right)$. Then the following a priori estimate holds

$$
\sup _{t \geq 0} \mathbf{E}\left(e^{-\beta t}\left|\widehat{X}_{t}\right|^{2}\right)+\left(\beta-2 \mu_{1}-2 L^{2}\right) \mathbf{E} \int_{0}^{+\infty} e^{-\beta t}\left|\widehat{X}_{s}\right|^{2} d s \leq\left|x_{1}-x_{2}\right|^{2},
$$

for $\beta>2 \mu_{1}+2 L^{2}$ and $\widehat{X}_{t}=X_{t}^{1}-X_{t}^{2}$. 
Proof: First assume that $\beta \in \mathbb{R}$ is arbitrary and fix some $t>0$. At the end of the proof, the right value of $\beta$ will be specified. Using Itô formula to $e^{-\beta t}\left|\widehat{X}_{t}\right|^{2}$ on $[0, t]$ one arrives at

$$
\begin{aligned}
e^{-\beta t}\left|\widehat{X}_{t}\right|^{2} & +\beta \int_{0}^{t} e^{-\beta s}\left|\widehat{X}_{s}\right|^{2} d s \\
& =\left|x_{1}-x_{2}\right|^{2}+2 \int_{0}^{t} e^{-\beta s}\left\langle\widehat{X}_{s}, b\left(X_{s}^{1}, u_{s}\right)-b\left(X_{s}^{2}, u_{s}\right)\right\rangle d s \\
& +2 \int_{0}^{t} e^{-\beta s}\left\langle\widehat{X}_{s},\left(\sigma\left(X_{s}^{1}, u_{s}\right)-\sigma\left(X_{s}^{2}, u_{s}\right)\right) d W_{s}\right\rangle \\
& +\int_{0}^{t} e^{-\beta s} \| \sigma\left(X_{s}^{1}, u_{s}\right)-\left.\sigma\left(X_{s}^{2}, u_{s}\right)\right|^{2} d s
\end{aligned}
$$

Now, taking $\mathbf{E}(\cdot)$ and employing $(\mathrm{H} 2)-(\mathrm{H} 3)$ we obtain

$$
\mathbf{E}\left(e^{-\beta t}\left|\widehat{X}_{t}\right|^{2}\right)+\left(\beta-2 \mu_{1}-2 L^{2}\right) \mathbf{E} \int_{0}^{t} e^{-\beta s}\left|\widehat{X}_{s}\right|^{2} d s \leq\left|x_{1}-x_{2}\right|^{2} .
$$

Then for $\beta>2 \mu_{1}+2 L^{2}$, we get 3.8.

Remark 1. One could easily obtain a similar estimate to (3.8) but with the supremum inside the expectation. In that case, we have to consider $\beta>2 \mu_{1}+2 L^{2}\left(C^{2}+1\right)$ where $C$ is the constant from Burkholder-Davis-Gundy inequality. For purposes of deriving the sufficient maximum principle, the estimation (3.8) is satisfactory.

Now, we will be interested in the solution to the BSDE 3.1. We assume that we already know the solution $X=X^{u(\cdot)} \in \mathbf{L}_{\mathcal{F}}^{2,-\beta}\left(\mathbb{R}_{+} ; \mathbb{R}^{n}\right)$ to SDE 3.2 for some fixed $u(\cdot) \in \mathcal{U}_{\text {ad }}$ assuming (H1)-(H3). The couple $(X, u)$ will be the (fixed) input for the BSDE 3.1.

We start the study of the backward equation by further specifying the coefficients $b, \sigma, f$. We assume that

$$
\begin{aligned}
& b: \mathbb{R}^{n} \times U \rightarrow \mathbb{R}^{n}, \\
& \sigma: \mathbb{R}^{n} \times U \rightarrow \mathbb{R}^{n \times d}, \\
& f: \mathbb{R}^{n} \times U \times \rightarrow \mathbb{R},
\end{aligned}
$$

such that

(H4): $b(x, u), \sigma(x, u)$ and $f(x, u)$ are continuously differentiable in $x$ for all $u \in U$.

(H5): There exists $\mu_{2} \in \mathbb{R}$ such that

$$
\left\langle y_{1}-y_{2}, \nabla_{x} b(x, u)\left(y_{1}-y_{2}\right)\right\rangle \leq \mu_{2}\left|y_{1}-y_{2}\right|^{2},
$$

for any $\left(x, u, y_{1}, y_{2}\right) \in \mathbb{R}^{n} \times U \times \mathbb{R}^{n} \times \mathbb{R}^{n}$.

(H6): There exists a constant $M \geq 0$ such that

$$
\left\|D_{x} \sigma(x, u)\right\|:=\sum_{i=1}^{d}\left\|\nabla_{x} \sigma^{i}(x, u)\right\| \leq M,
$$

for any $(x, u) \in \mathbb{R}^{n} \times U$.

Using the transform $Y_{t}=e^{\beta t} \widetilde{Y}_{t}$ and $Z_{t}=e^{\beta t} \widetilde{Z}_{t}$, the infinite horizon BSDE (3.1) can be rewritten as

$$
-d \widetilde{Y}_{t}=\left(\nabla_{x} b\left(X_{t}, u_{t}\right) \widetilde{Y}_{t}+D_{x} \sigma\left(X_{t}, u_{t}\right) \cdot \widetilde{Z}_{t}+e^{-\beta t} \nabla_{x} f\left(X_{t}, u_{t}\right)\right) d t-\widetilde{Z}_{t} d W_{t}, \quad \forall t \geq 0,
$$

If we show that the solution process $(\tilde{Y}, \widetilde{Z})$ is in $\mathbf{L}_{\mathcal{F}}^{2, \beta}\left(\mathbb{R}_{+} ; \mathbb{R}^{n} \times \mathbb{R}^{n \times d}\right)$ then necessarily the original solution process $(Y, Z)$ has to be in $\mathbf{L}_{\mathcal{F}}^{2,-\beta}\left(\mathbb{R}_{+} ; \mathbb{R}^{n} \times \mathbb{R}^{n \times d}\right)$ since we have

$$
\mathbf{E} \int_{0}^{+\infty} e^{-\beta t}\left|Y_{t}\right|^{2} d t=\mathbf{E} \int_{0}^{+\infty} e^{-\beta t}\left|e^{\beta t} \widetilde{Y}_{t}\right|^{2} d t=\mathbf{E} \int_{0}^{+\infty} e^{\beta t}\left|\widetilde{Y}_{t}\right|^{2} d t<+\infty,
$$

and similarly for the process $Z$. 
The existence and uniqueness is given by Theorem 4 in Peng and Shi [23]. In our setting, it is formulated as follows:

Theorem 2. [Existence and uniqueness] Let assumptions (H4)-(H6) hold and $\left(X_{t}^{u(\cdot)}, u(\cdot)\right)$ be an admissible couple such that $X_{t}^{u(\cdot)} \in \mathbf{L}_{\mathcal{F}}^{2,-\beta}\left(\mathbb{R}_{+} ; \mathbb{R}^{n}\right)$ and $\nabla_{x} f\left(X_{t}, u_{t}\right) \in \mathbf{L}_{\mathcal{F}}^{2,-\beta}\left(\mathbb{R}_{+} ; \mathbb{R}^{n}\right)$ where $\beta>2 \mu_{2}+2 M^{2}$. Then BSDE (3.13) admits a unique solution $(\widetilde{Y}, \widetilde{Z}) \in \mathbf{L}_{\mathcal{F}}^{2, \beta}\left(\mathbb{R}_{+} ; \mathbb{R}^{n} \times \mathbb{R}^{n \times d}\right)$.

Remark 2. The sign of $\beta$ depends on the sign of the factor $2 \mu_{2}+2 M^{2}$. In case of $\mu_{2}<0$ one gets so called weak monotonicity condition as in Peng, Shi [23] allowing also $\beta \leq 0$. As we will see later in Example 3, the controlled logistic equation naturally leads to $\mu_{2}>0$ which implies $\beta>0$.

Using the previous theorem and 3.14 we conclude this section by

Corollary 3. Let assumptions (H1)-(H6) hold and suppose that $\nabla_{x} f\left(X_{t}, u_{t}\right) \in \mathbf{L}_{\mathcal{F}}^{2,-\beta}\left(\mathbb{R}_{+} ; \mathbb{R}^{n}\right)$. Then BSDE (3.1) admits a unique solution $(Y, Z) \in \mathbf{L}_{\mathcal{F}}^{2,-\beta}\left(\mathbb{R}_{+} ; \mathbb{R}^{n} \times \mathbb{R}^{n \times d}\right)$ for $\beta>2 \mu_{2}+2 M^{2}$.

Remark 3. The above theorems stand valid if the assumptions (H1)-(H6) are fulfilled for all $x \in$ $G \subset \mathbb{R}^{n}, G$ domain, provided $X_{t} \in G$ for all $t \geq 0$ a.s.

3.1. Stability of BSDE under approximations. In this subsection we legitimate the approximation the BSDE by an equation with terminal zero condition. As we have already mentioned when dealing with DCP, the terminal value $\xi$ of the solution process $Y$ is not known. We will show that under (H1)-(H6) and natural assumptions on $\xi$ (finite second moment) the approximation is stable, i.e. one can choose zero terminal condition or $\mathcal{F}_{t}$ - measurable its projections to obtain the same solution process. The following lemma connects the approach in Darling and Pardoux [20] and in Peng and Shi [23].

Lemma 2. [Stability under approximations] Let (H1)-(H6) hold and let $\xi$ be an $\mathbb{R}^{n}$-valued $\mathcal{F}_{\infty}$ - measurable random variable with $\mathbf{E}|\xi|^{2}<+\infty$. Further, for each $n \in \mathbb{N}$, let us consider two $B S D E$ 's

$$
\begin{aligned}
-d \widetilde{Y}_{t}^{n} & =\nabla_{x} \mathcal{H}\left(X_{t}, u_{t}, \widetilde{Y}_{t}^{n}, \widetilde{Z}_{t}^{n}\right) d t-\widetilde{Z}_{t}^{n} d W_{t}, t \in[0, n) \\
\widetilde{Y}_{n}^{n} & =0,
\end{aligned}
$$

and

$$
\begin{aligned}
-d \widehat{Y}_{t}^{n} & =\nabla_{x} \mathcal{H}\left(X_{t}, u_{t}, \widehat{Y}_{t}^{n}, \widehat{Z}_{t}^{n}\right) d t-\widehat{Z}_{t}^{n} d W_{t}, t \in[0, n) \\
\widehat{Y}_{n}^{n} & =\xi_{n}:=\mathbf{E}\left[\xi \mid \mathcal{F}_{n}\right],
\end{aligned}
$$

where $\nabla_{x} \mathcal{H}(x, u, y, z)=\nabla_{x} b(x, u) y+D_{x} \sigma(x, u) \cdot z+\nabla_{x} f(x, u)-\beta y$.

We lay $\widetilde{Y}_{t}^{n}=0$ and $\widehat{Y}_{t}^{n}=\xi_{t}:=\mathbf{E}\left[\xi \mid \mathcal{F}_{t}\right], \forall t>n$.

Then

$$
\begin{aligned}
\lim _{n \rightarrow+\infty}\left[\sup _{t \geq 0} \mathbf{E}\left(e^{-\beta t}\left|\widetilde{Y}_{t}^{n}-\widehat{Y}_{t}^{n}\right|^{2}\right)\right. & +\mathbf{E} \int_{0}^{+\infty} \mathbf{1}_{[0, n]}(t) e^{-\beta t}\left|\widetilde{Y}_{t}^{n}-\widehat{Y}_{t}^{n}\right|^{2} d t \\
& \left.+\mathbf{E} \int_{0}^{+\infty} \mathbf{1}_{[0, n]}(t) e^{-\beta t}\left\|\widetilde{Z}_{t}^{n}-\widehat{Z}_{t}^{n}\right\|^{2} d t\right]=0,
\end{aligned}
$$

for $\beta \geq 2 \mu_{2}+2 M^{2}>0$.

Proof: First observe that, using Jensen's inequality and integrability of $\xi$, one gets

$$
\mathbf{E}\left(e^{-\beta n}\left|\xi_{n}\right|^{2}\right)=e^{-\beta n} \mathbf{E}\left(\left|\mathbf{E}\left(\xi \mid \mathcal{F}_{n}\right)\right|^{2}\right) \leq \mathbf{E}\left(e^{-\beta n}|\xi|^{2}\right) \underset{n \rightarrow+\infty}{\rightarrow} 0 .
$$

Now, applying Itô formula to $e^{-\beta t}\left|\widetilde{Y}_{t}^{n}-\widehat{Y}_{t}^{n}\right|^{2}$ one arrives at

$$
\begin{aligned}
& \mathbf{E}\left(e^{-\beta t}\left|\widetilde{Y}_{t}^{n}-\widehat{Y}_{t}^{n}\right|^{2}\right)+\beta \mathbf{E} \int_{0}^{n} e^{-\beta s}\left|\widetilde{Y}_{s}^{n}-\widehat{Y}_{s}^{n}\right|^{2} d s+\mathbf{E} \int_{0}^{n} e^{-\beta s} \| \widetilde{Z}_{s}^{n}-\widehat{Z}_{s}^{n}||^{2} d s=\mathbf{E}\left(e^{-\beta n}\left|\xi_{n}\right|^{2}\right) \\
& \quad+2 \mathbf{E} \int_{0}^{n} e^{-\beta s}\left[\left\langle\nabla_{x} b\left(X_{s}, u_{s}\right)\left(\widetilde{Y}_{s}^{n}-\widehat{Y}_{s}^{n}\right), \widetilde{Y}_{s}^{n}-\widehat{Y}_{s}^{n}\right\rangle+\left\langle D_{x} \sigma\left(X_{s}, u_{s}\right) \cdot\left(\widetilde{Z}_{s}^{n}-\widehat{Z}_{s}^{n}\right), \widetilde{Y}_{s}^{n}-\widehat{Y}_{s}^{n}\right\rangle\right] d s .
\end{aligned}
$$


Now, due to (H5) and (H6), we arrive at

$$
\begin{aligned}
\mathbf{E}\left(e^{-\beta t}\left|\widetilde{Y}_{t}^{n}-\widehat{Y}_{t}^{n}\right|^{2}\right) & +\left(\beta-2 \mu_{2}-2 M^{2}\right) \mathbf{E} \int_{0}^{n} e^{-\beta s}\left|\widetilde{Y}_{s}^{n}-\widehat{Y}_{s}^{n}\right|^{2} d s \\
& +\frac{1}{2} \mathbf{E} \int_{0}^{n} e^{-\beta s}|| \widetilde{Z}_{s}^{n}-\widehat{Z}_{s}^{n} \|^{2} d s \leq \mathbf{E}\left(e^{-\beta n}\left|\xi_{n}\right|^{2}\right), \forall 0 \leq t \leq n
\end{aligned}
$$

Taking the $\sup _{0 \leq t \leq n}$ on both sides of 3.20 , we obtain

$$
\begin{aligned}
\sup _{0 \leq t \leq n} \mathbf{E}\left(e^{-\beta t}\left|\widetilde{Y}_{t}^{n}-\widehat{Y}_{t}^{n}\right|^{2}\right) & +\left(\beta-2 \mu_{2}-2 M^{2}\right) \mathbf{E} \int_{0}^{n} e^{-\beta s}\left|\widetilde{Y}_{s}^{n}-\widehat{Y}_{s}^{n}\right|^{2} d s \\
& +\frac{1}{2} \mathbf{E} \int_{0}^{n} e^{-\beta s}|| \widetilde{Z}_{s}^{n}-\widehat{Z}_{s}^{n} \|^{2} d s \leq \mathbf{E}\left(e^{-\beta n}\left|\xi_{n}\right|^{2}\right) \underset{n \rightarrow+\infty}{\rightarrow} 0 .
\end{aligned}
$$

To obtain the estimate for all $t \geq 0$ and for $\beta \geq 2 \mu_{2}+2 M^{2}$ we write

$$
\begin{gathered}
\sup _{t \geq 0} \mathbf{E}\left(e^{-\beta t}\left|\tilde{Y}_{t}^{n}-\widehat{Y}_{t}^{n}\right|^{2}\right) \leq \sup _{0 \leq t \leq n} \mathbf{E}\left(e^{-\beta t}\left|\widetilde{Y}_{t}^{n}-\widehat{Y}_{t}^{n}\right|^{2}\right)+\sup _{t>n} \mathbf{E}\left(e^{-\beta t}\left|\widetilde{Y}_{t}^{n}-\widehat{Y}_{t}^{n}\right|^{2}\right) \\
\quad \leq \mathbf{E}\left(e^{-\beta n}\left|\xi_{n}\right|^{2}\right)+\sup _{t>n} \mathbf{E}\left(e^{-\beta t}\left|\xi_{t}\right|^{2}\right) \underset{n \rightarrow+\infty}{\rightarrow} 0 .
\end{gathered}
$$

The last term is due to definition of $\widetilde{Y}^{n}$ and $\widehat{Y}^{n}$ for $t \geq 0$ and due to 3.18. The convergence of the integral terms in 3.17 follows easily.

Remark 4. In the above Lemma 2 we could impose even more general conditions on $\xi$ ensuring $\mathbf{E}\left(e^{-\beta n}\left|\xi_{n}\right|^{2}\right) \rightarrow 0$ as $n \rightarrow+\infty$.

\section{SUfFiCIENT STOCHASTIC MAXIMUM PRINCIPLE FOR THE DCP}

In this section, we return to the discounted control problem and prove the corresponding sufficient maximum principle. First, we employ the approach using the results from the previous section. Second, we apply results from $\emptyset \mathrm{ksendal}$ et al. [12] to our definition of Hamiltonian and prove the associated DCP using transversality condition. Finally, the connection between the two methods is shown.

Theorem 4. [Sufficient stochastic maximum principle] Let (H1) - (H6) hold and $\beta>\max \left\{2 \mu_{1}+2 L^{2}, 2 \mu_{2}+2 M^{2}\right\}$. Assume moreover that $\nabla_{x} f\left(X_{t}, u_{t}\right) \in \mathbf{L}_{\mathcal{F}}^{2,-\beta}\left(\mathbb{R}_{+} ; \mathbb{R}^{n}\right)$ for any admissible couple $(X, u)$ from Theorem $\square$ Further, let $\widehat{u}(\cdot) \in \mathcal{U}_{a d}$ and $\widehat{X}$ be the associated controlled diffusion process. Let us suppose that there exists a solution $(\widehat{Y}, \widehat{Z})$ to the associated BSDE (3.1) such that

(1) $\mathcal{H}\left(\widehat{X}_{t}, \widehat{u}_{t}, \widehat{Y}_{t}, \widehat{Z}_{t}\right)=\max _{u \in U} \mathcal{H}\left(\widehat{X}_{t}, u, \widehat{Y}_{t}, \widehat{Z}_{t}\right), \mathbf{P} \otimes d t-$ a.e.,

(2) $(x, u) \rightarrow \mathcal{H}\left(x, u, \widehat{Y}_{t}, \widehat{Z}_{t}\right)$ is a concave function, $\mathbf{P} \otimes d t-$ a.e.

Then $\widehat{u}(\cdot)=u^{*}(\cdot)$, i.e. $\widehat{u}(\cdot)$ is the optimal control strategy to the DCP.

Proof: Take an arbitrary $u(\cdot) \in \mathcal{U}_{a d}$ and examine the difference $J(\widehat{u}(\cdot))-J(u(\cdot))$. The goal is to show that this quantity is nonnegative. Using the definition of $J(u(\cdot))$ and $\mathcal{H}$ we have

$$
\begin{aligned}
& J(\widehat{u}(\cdot))-J(u(\cdot))=\mathbf{E} \int_{0}^{+\infty} e^{-\beta t}\left(f\left(\widehat{X}_{t}, \widehat{u}_{t}\right)-f\left(X_{t}, u_{t}\right)\right) d t \\
& =\mathbf{E} \int_{0}^{+\infty} e^{-\beta t}\left(\mathcal{H}\left(\widehat{X}_{t}, \widehat{u}_{t}, \widehat{Y}_{t}, \widehat{Z}_{t}\right)-\mathcal{H}\left(X_{t}, u_{t}, \widehat{Y}_{t}, \widehat{Z}_{t}\right)+\left\langle b\left(X_{t}, u_{t}\right)-b\left(\widehat{X}_{t}, \widehat{u}_{t}\right), \widehat{Y}_{t}\right\rangle\right. \\
& \left.\quad+\operatorname{Tr}\left\{\left(\sigma^{\prime}\left(X_{t}, u_{t}\right)-\sigma^{\prime}\left(\widehat{X}_{t}, \widehat{u}_{t}\right)\right) \widehat{Z}_{t}\right\}+\beta\left\langle\widehat{X}_{t}-X_{t}, \widehat{Y}_{t}\right\rangle\right) d t
\end{aligned}
$$

Clearly, the r.h.s. of 4.1 is finite hence we obtain

$$
\mathbf{E} \int_{0}^{+\infty} \mathcal{I}_{t} d t=\lim _{T \rightarrow+\infty} \mathbf{E} \int_{0}^{T} \mathcal{I}_{t} d t,
$$


where $\mathcal{I}_{t}$ is the integrand of 4.1.

Further, since (H1)-(H6) hold we know by Corollary 3 that each of the three solution processes $X, \widehat{X}, \widehat{Y}$ belongs to $\mathbf{L}_{\mathcal{F}}^{2,-\beta}\left(\mathbb{R}_{+} ; \mathbb{R}^{n}\right)$ and therefore there exists a sequence $\left(T_{n}\right)_{n \in \mathbb{N}}, T_{n} \nearrow+\infty$ as $n \rightarrow+\infty$ so that

$$
\left|\mathbf{E}\left(e^{-\beta T_{n}}\left\langle\widehat{X}_{T_{n}}-X_{T_{n}}, \widehat{Y}_{T_{n}}\right\rangle\right)\right| \leq \frac{1}{2} \mathbf{E}\left(e^{-\beta T_{n}}\left|\widehat{X}_{T_{n}}-X_{T_{n}}\right|^{2}\right)+\frac{1}{2} \mathbf{E}\left(e^{-\beta T_{n}}\left|\widehat{Y}_{T_{n}}\right|^{2}\right)_{n \rightarrow+\infty}^{\rightarrow} 0,
$$

where we have applied Cauchy-Schwarz inequality and the Young inequality $2 a b \leq \frac{1}{2} a^{2}+\frac{1}{2} b^{2}$, $a, b \in \mathbb{R}$. By (4.2) and (4.3) we have that

$$
J(\widehat{u}(\cdot))-J(u(\cdot))=\lim _{n \rightarrow+\infty} \mathbf{E}\left[\int_{0}^{T_{n}} \mathcal{I}_{t} d t+e^{-\beta T_{n}}\left\langle\widehat{X}_{T_{n}}-X_{T_{n}}, \widehat{Y}_{T_{n}}\right\rangle\right] .
$$

Now, applying Itô formula to the last term in the bracket and taking $\mathbf{E}(\cdot)$ we arrive to

$$
\begin{aligned}
& \mathbf{E}\left[e^{-\beta T_{n}}\left\langle\widehat{X}_{T_{n}}-X_{T_{n}}, \widehat{Y}_{T_{n}}\right\rangle\right]=\mathbf{E} \int_{0}^{T_{n}} e^{-\beta t}\left(\left\langle b\left(\widehat{X}_{t}, \widehat{u}_{t}\right)-b\left(X_{t}, u_{t}\right), \widehat{Y}_{t}\right\rangle\right. \\
&+\operatorname{Tr}\left\{\left(\sigma^{\prime}\left(\widehat{X}_{t}, \widehat{u}_{t}\right)-\sigma^{\prime}\left(X_{t}, u_{t}\right)\right) \widehat{Z}_{t}\right\}-\beta\left\langle\widehat{X}_{t}-X_{t}, \widehat{Y}_{t}\right\rangle \\
&\left.-\left\langle\widehat{X}_{t}-X_{t}, \nabla_{x} \mathcal{H}\left(\widehat{X}_{t}, \widehat{u}_{t}, \widehat{Y}_{t}, \widehat{Z}_{t}\right)\right\rangle\right) d t
\end{aligned}
$$

In view of the equality (4.4), we finally arrive at

$$
\begin{gathered}
J(\widehat{u}(\cdot))-J(u(\cdot))=\lim _{n \rightarrow+\infty} \mathbf{E} \int_{0}^{T_{n}} e^{-\beta t}\left(\mathcal{H}\left(\widehat{X}_{t}, \widehat{u}_{t}, \widehat{Y}_{t}, \widehat{Z}_{t}\right)-\mathcal{H}\left(X_{t}, u_{t}, \widehat{Y}_{t}, \widehat{Z}_{t}\right)\right. \\
\left.-\left\langle\widehat{X}_{t}-X_{t}, \nabla_{x} \mathcal{H}\left(\widehat{X}_{t}, \widehat{u}_{t}, \widehat{Y}_{t}, \widehat{Z}_{t}\right)\right\rangle\right) d t .
\end{gathered}
$$

By the concavity of $\mathcal{H}$ in $(x, u)$, we have that

$$
\mathcal{H}\left(\widehat{X}_{t}, \widehat{u}_{t}, \widehat{Y}_{t}, \widehat{Z}_{t}\right)-\mathcal{H}\left(X_{t}, u_{t}, \widehat{Y}_{t}, \widehat{Z}_{t}\right)-\left\langle\widehat{X}_{t}-X_{t}, \nabla_{x} \mathcal{H}\left(\widehat{X}_{t}, \widehat{u}_{t}, \widehat{Y}_{t}, \widehat{Z}_{t}\right)\right\rangle \geq 0
$$

Therefore, we deduce that

$$
J(\widehat{u}(\cdot))-J(u(\cdot)) \geq 0, \quad \forall u(\cdot) \in \mathcal{U}_{a d},
$$

which proves that $\widehat{u}(\cdot)$ is indeed the optimal control.

Now, we provide a similar version of sufficient maximum principle proved in [12], Theorem 4.1 using the so called transversality condition (TVC). The fact which may be inconvenient for the controller is that the TVC has to be verified (theoretically) for every admissible control $u(\cdot)$. Moreover, this criterion, in general, cannot be explicitly verified in terms of the coefficients $b, \sigma, f$ and $\beta$ of the DCP. On the other hand, TVC has an economical interpretation for the DCP, namely it expresses the additional benefit of one unit of good for time increasing to infinity.

Theorem 5. [Sufficient stochastic maximum principle] Let $\widehat{u}(\cdot) \in \mathcal{U}_{\text {ad }}$ and $\widehat{X}$ be the associated controlled diffusion process. Let us suppose that there exists a solution $(\widehat{Y}, \widehat{Z})$ to the associated BSDE (3.1) such that

(1) $\mathcal{H}\left(\widehat{X}_{t}, \widehat{u}_{t}, \widehat{Y}_{t}, \widehat{Z}_{t}\right)=\max _{u \in U} \mathcal{H}\left(\widehat{X}_{t}, u, \widehat{Y}_{t}, \widehat{Z}_{t}\right), \quad \mathbf{P} \otimes d t-$ a.e.,

(2) $(x, u) \rightarrow \mathcal{H}\left(x, u, \widehat{Y}_{t}, \widehat{Z}_{t}\right)$ is a concave function, $\quad \mathbf{P} \otimes d t-$ a.e.,

(3) the transversality condition (TVC)

$$
\varlimsup_{t \rightarrow+\infty} \mathbf{E}\left(e^{-\beta t}\left\langle\widehat{X}_{t}-X_{t}, \widehat{Y}_{t}\right\rangle\right) \leq 0
$$

holds for every $X=X^{u(\cdot)}, u(\cdot) \in \mathcal{U}_{a d}$.

Then $\widehat{u}(\cdot)=u^{*}(\cdot)$, i.e. $\widehat{u}(\cdot)$ is the optimal control strategy to the DCP. 
Remark 5. [Sufficient condition for TVC] We will show that the conditions (H1)-(H6) immediately imply the TVC, which provides an easy-to-verify approach for the controller.

Indeed, we have shown that under (H1)-(H6), the solution processes to the FBSDE (3.1)

$$
\begin{aligned}
(X, Y, Z) & =\left(X^{x, u(\cdot)}, Y^{x, u(\cdot), X}, Z^{x, u(\cdot), X}\right) \text { and } \\
(\widehat{X}, \widehat{Y}, \widehat{Z}) & =\left(\widehat{X}^{x, \widehat{u}(\cdot)}, \widehat{Y}^{x, \widehat{u}(\cdot), \widehat{X}}, \widehat{Z}^{x, \widehat{u}(\cdot), \widehat{X}}\right),
\end{aligned}
$$

are both in $\mathbf{L}_{\mathcal{F}}^{2,-\beta}\left(\mathbb{R}_{+} ; \mathbb{R}^{n} \times \mathbb{R}^{n} \times \mathbb{R}^{n \times d}\right)$ for $\beta>\max \left\{2 \mu_{1}+2 L^{2}, 2 \mu_{2}+2 M^{2}\right\}$.

Therefore, there exists a sequence $\left(T_{n}\right)_{n \in \mathbb{N}}, T_{n} \nearrow+\infty$ as $n \rightarrow+\infty$ so that

$$
\left|\mathbf{E}\left(e^{-\beta T_{n}}\left\langle\widehat{X}_{T_{n}}-X_{T_{n}}, \widehat{Y}_{T_{n}}\right\rangle\right)\right| \leq \frac{1}{2} \mathbf{E}\left(e^{-\beta T_{n}}\left|\widehat{X}_{T_{n}}-X_{T_{n}}\right|^{2}\right)+\frac{1}{2} \mathbf{E}\left(e^{-\beta T_{n}}\left|\widehat{Y}_{T_{n}}\right|^{2}\right) \underset{n \rightarrow+\infty}{\rightarrow} 0,
$$

which implies the TVC 4.8.

Remark 6. All results of section 4 holds also for time dependent coefficients $b: \mathbb{R}_{+} \times \mathbb{R}^{n} \times U \rightarrow$ $\mathbb{R}^{n}, \sigma: \mathbb{R}_{+} \times \mathbb{R}^{n} \times U \rightarrow \mathbb{R}^{n \times d}, f: \mathbb{R}_{+} \times \mathbb{R}^{n} \times U \rightarrow \mathbb{R}$ under natural assumptions.

Remark 7. [Hamiltonian system] Throughout the paper we assume that the Hamiltonian takes the form (2.7). Nevertheless, one could easily verify that Theorem 4 holds also with the Hamiltonian function $\mathcal{H}$ replaced by a different Hamiltonian function $H$ defined as

$$
H(x, u, y, z)=\langle b(x, u), y\rangle+\operatorname{Tr}\left(\sigma(x, u)^{\prime} z\right)+f(x, u)=\mathcal{H}(x, u, y, z)+\beta\langle x, y\rangle
$$

which enables us to rewrite the Forward-Backward Hamiltonian system as

$$
\begin{aligned}
d X_{t} & =\nabla_{y} H\left(X_{t}, u_{t}, Y_{t}, Z_{t}\right) d t+\nabla_{z} H\left(X_{t}, u_{t}, Y_{t}, Z_{t}\right) d W_{t}, \quad \forall t \geq 0 \text { a.s. } \\
X_{0} & =x \in \mathbb{R}^{n} \\
-d Y_{t} & =\left(\nabla_{x} H\left(X_{t}, u_{t}, Y_{t}, Z_{t}\right)-\beta Y_{t}\right) d t-Z_{t} d W_{t}, \quad \forall t \geq 0, \text { a.s. }
\end{aligned}
$$

Again, there is a correction term one has take into consideration, namely $-\beta Y_{t}$ in the driver of the $B S D E$.

\section{EXAMPLES}

In this section we provide two illustrative examples of discounted control problems with well known solution. It will be shown that the approach using our maximum principle lead to the solution as well.

5.1. Example 1 - Production planning problem. The setting of the problem is taken from Borkar [6] and it is in fact the classical LQ problem whose solution is well known from e.g. dynamic programming, see Øksendal [15], chapter 11. In the feedback Markov setting, the optimal control is obtained in the form of the observed (Markov) diffusion state process $X_{t}$ plugged into the solution of the associated Riccati ODE.

Let us consider a factory producing a single item. Let $X(\cdot)$ denote its inventory level as a function of time and $u(\cdot) \geq 0$ denote the production rate. $\eta$ denotes the constant demand rate and $x_{1}, u_{1}$ resp. the factory-optimal inventory level and production rate.

The inventory process is modeled as the controlled diffusion

$$
d X_{t}=\left(u_{t}-\eta\right) d t+\sigma d W_{t}, \quad \forall t \geq 0,
$$

where $\sigma$ is a constant. The aim is to minimize over non-anticipative $u(\cdot)$ the discounted cost

$$
J(u(\cdot))=\mathbf{E} \int_{0}^{+\infty} e^{-\beta t}\left(c\left(u_{t}-u_{1}\right)^{2}+h\left(X_{t}-x_{1}\right)^{2}\right) d t,
$$

where $c, h>0$ are known coefficients for the production cost and the inventory holding cost, resp. and $\beta>0$ is a discount factor. 
The minimization task is converted to maximization by taking $-J(u(\cdot))$ as the functional. Firstly, it is easy to see that (H1)-(H6) hold for $\mu_{1}=\mu_{2}=L=M=0$ and therefore the DCP is meaningful for all $\beta \geq 0$. The Hamiltonian of this control problem is

$$
\mathcal{H}(x, u, y, z)=(u-\eta) y+\sigma z-c\left(u-u_{1}\right)^{2}-h\left(x-x_{1}\right)^{2}-\beta x y
$$

which is a concave function in $(x, u)$. The driver of the backward adjoint equation is obtained as the derivative of $\mathcal{H}$ w.r.t $x$, i.e.

$$
h(x, u, y, z)=\frac{\partial}{\partial x} \mathcal{H}(x, u, y, z)=-2 h\left(x-x_{1}\right)-\beta y
$$

and the associated BSDE is

$$
-d Y_{t}=\left(-2 h\left(X_{t}-x_{1}\right)-\beta Y_{t}\right) d t-Z_{t} d W_{t}, \quad \forall t \geq 0, \text { a.s. }
$$

To find the extremal (maximal) point of $\mathcal{H}$ we lay

which leads to

$$
\frac{\partial}{\partial u} \mathcal{H}(x, u, y, z)=y-2 c\left(u-u_{1}\right)=0
$$

$$
\widehat{u}_{t}=\frac{\widehat{Y}_{t}}{2 c}+u_{1} .
$$

Further, assuming the solution to BSDE $\underline{(5.3)}$ in the feedback form

$$
Y_{t}=\varphi_{t} X_{t}+\psi_{t}, \quad \forall t \geq 0 \text { a.s. }
$$

for some deterministic functions $\varphi, \psi$ in $\mathcal{C}^{1}$ one can arrive to Riccati system of ODE's similarly as in [17].

5.2. Example 2 - Optimal consumption rate. The problem of optimal consumption rate is taken from Øksendal [12] where it is solved using a different definition of Hamiltonian. In our setting, we can, in fact, follow exactly the solution procedure step by step showing the same result. The main difference is that using our approach, one immediately knows whether the transversality condition holds just by examining the coefficients.

Consider an agent whose wealth evolves according to the following controlled bilinear SDE in $\mathbb{R}$

$$
\begin{aligned}
d X_{t} & =X_{t}\left(\mu-u_{t}\right) d t+\sigma X_{t} d W_{t}, \quad \forall t \geq 0, \\
X_{0} & =x_{0}>0,
\end{aligned}
$$

where $u(\cdot)$ is the consumption rate process, $\mu$ and $\sigma$ are some real constants (unlike in [12] where they are assumed time dependent and bounded). The aim is to maximize over all strictly positive controls $u(\cdot)$ bounded by some constant $K>0$ the discounted cost functional

$$
J(u(\cdot))=\mathbf{E} \int_{0}^{+\infty} e^{-\beta t} \ln \left(u_{t} X_{t}\right) d t,
$$

First we see that (H1)-(H6) hold for $\mu_{1}=\mu_{2}=\mu, M=L=|\sigma|$. Further, one has to verify that the following condition holds

$$
\mathbf{E} \int_{0}^{+\infty} e^{-\beta t}\left\|\nabla_{x} f\left(X_{t}, u_{t}\right)\right\|^{2} d t<+\infty .
$$

To see it, realize that the solution to 5.6 is a geometric Brownian motion

$$
X_{t}=x_{0} \exp \left(\sigma W_{t}-\frac{1}{2} \sigma^{2} t+\int_{0}^{t}\left(\mu-u_{s}\right) d s\right),
$$

so the condition reads

$$
\mathbf{E} \int_{0}^{+\infty} e^{-\beta t} \frac{1}{\left|X_{t}\right|^{2}} d t<+\infty
$$

Since we have 


$$
\begin{aligned}
\mathbf{E}\left[\frac{1}{\left|X_{t}\right|^{2}}\right]=x_{0}^{-2} \mathbf{E}[\exp & \left.\left(\sigma^{2} t-2 \int_{0}^{t}\left(\mu-u_{s}\right) d s\right) \exp \left(-2 \sigma W_{t}\right)\right] \\
& \leq x_{0}^{-2} \exp \left\{\left(3 \sigma^{2}-2 \mu+K\right) t\right\}<+\infty,
\end{aligned}
$$

Theorem 4 may be applied for all $\beta>\max \left\{2 \mu+2 \sigma^{2}, K+3 \sigma^{2}-2 \mu\right\}$.

The Hamiltonian of this control problem is

$$
\mathcal{H}(x, u, y, z)=x(\mu-u) y+x \sigma z+\ln (x u)-\beta x y
$$

which is a concave function in $(x, u)$. The driver of the backward adjoint equation is obtained as the derivative of $\mathcal{H}$ w.r.t. $x$, i.e.

$$
\frac{\partial}{\partial x} \mathcal{H}(x, u, y, z)=(\mu-u-\beta) y+\sigma z+\frac{1}{x} .
$$

To find the maximal point of $\mathcal{H}$ we lay

which leads to

$$
\frac{\partial}{\partial u} \mathcal{H}(x, u, y, z)=-x y+\frac{1}{u}=0,
$$

$$
\widehat{u}_{t}=\frac{1}{\widehat{X}_{t} \widehat{Y}_{t}}
$$

and the associated BSDE is

$$
-d \widehat{Y}_{t}=\left(\left(\mu-\widehat{u}_{t}-\beta\right) \widehat{Y}_{t}+\sigma \widehat{Z}_{t}+\frac{1}{\widehat{X}_{t}}\right) d t-\widehat{Z}_{t} d W_{t}, \quad \forall t \geq 0 .
$$

The solution to BSDE (5.14) for a general admissible control $u(\cdot)$ can be find along similar lines as in [12]. We obtain

$$
Y_{t}=\frac{1}{X_{t} \beta}
$$

Finally, using 5.13 and 5.15 the optimal control is

$$
\widehat{u}_{t}=\beta \text {. }
$$

\section{Controlled Stochastic logistic EQuation}

In this section we assume that the controlled dynamics is given by a stochastic differential equation with controlled logistic equation as a special case. It provides an example of a system with concave nonlinear coefficients. Here, the DCP is defined as follows. A one dimensional controlled state dynamics is given by

$$
\begin{aligned}
d X_{t} & =X_{t} F\left(X_{t}, u_{t}\right) d t+\sigma\left(X_{t}\right) d W_{t}, \quad \forall t \geq 0, \\
X_{0} & =x_{0},
\end{aligned}
$$

where $u(\cdot)$ is a control process with values in $U=[\underline{U}, \bar{U}] \times[\underline{V}, \bar{V}] \subset \mathbb{R}_{+}^{2}=[0,+\infty)^{2}$ and $x_{0}>0$ is a deterministic initial value. The functions $F: \mathbb{R} \times U \rightarrow \mathbb{R}$ and $\sigma: \mathbb{R} \rightarrow \mathbb{R}$ are continuously differentiable in $x, F$ is continuous in $(x, u)$, functions $b(x, u)=x F(x, u)$ and $\sigma(x)$ are concave in all their variables and we further assume that

- $\sigma(0)=0$,

- $\sigma(\cdot)$ is globally Lipschitz, i.e. there exists $L>0$ constant such that

$$
\left|\sigma\left(x_{1}\right)-\sigma\left(x_{2}\right)\right| \leq L\left|x_{1}-x_{2}\right|, \forall\left(x_{1}, x_{2}\right) \in \mathbb{R}^{2},
$$

- there exists $M>0$ constant such that $\left|\sigma^{\prime}(x)\right| \leq M, \forall x \in \mathbb{R}$, 
- there exists $\mu_{1} \in \mathbb{R}$ such that

$$
\left(x_{1}-x_{2}\right)\left(x_{1} F\left(x_{1}, u\right)-x_{2} F\left(x_{2}, u\right)\right) \leq \mu_{1}\left|x_{1}-x_{2}\right|^{2}, \forall x_{1}, x_{2} \geq 0, u \in U,
$$

- there exists $\mu_{2} \in \mathbb{R}$ such that

$$
\left(y_{1}-y_{2}\right)^{2}\left(F(x, u)+x \frac{\partial}{\partial x} F(x, u)\right) \leq \mu_{2}\left|y_{1}-y_{2}\right|^{2},
$$

$\forall x>0, u \in U,\left(y_{1}, y_{2}\right) \in \mathbb{R}^{2}$.

- there exist constants $r, R, C>0$ such that

$$
\begin{aligned}
-F(x, u) & \leq C, \forall x \in(0, r), u \in U, \\
F(x, u) & \leq C, \forall x \in(R,+\infty), u \in U .
\end{aligned}
$$

Notice that the two functions $b(x, u)=x F(x, u)$ and $\sigma(x)$ fulfil the assumptions (H1) - (H6). Assume further that

$$
x F(x,(\underline{U}, \bar{V})) \leq x F(x, u) \leq x F(x,(\bar{U}, \underline{V})), \forall x>0, \forall u \in U,
$$

and denote the corresponding (constant) controls as $u_{\text {lower }}=(\underline{U}, \bar{V})$ and $u_{\text {upper }}=(\bar{U}, \underline{V})$.

Note that the notation $F(\cdot, \cdot)$ in the drift in equation 6.1 covers situations of controlled drift such as: $x F(x, u)=a x\left(u_{1}-b u_{2} x\right)$ for $u=\left(u_{1}, u_{2}\right)^{\prime}$, in which one can recognize the logistic equation with controlled both the saturation level of the diffusion and the speed of reaching this level.

The set of admissible controls $\mathcal{U}_{a d}$ is defined as

$$
\mathcal{U}_{a d}=\left\{u: \mathbb{R}_{+} \times \Omega \rightarrow U: u \in \mathbf{L}_{\mathcal{F}, l o c}^{2}(0,+\infty ; U)\right\}
$$

The functional to be minimized over all admissible controls $u(\cdot)$ is

$$
J(u(\cdot))=\mathbf{E} \int_{0}^{+\infty} e^{-\beta t}\left(c X_{t}^{2}+h\left|u_{t}\right|^{2}\right) d t,
$$

where $c, h>0$ and $\beta>0$ is a discount factor.

First we start by showing that the solution to 6.1 for $x_{0}>0$ stays in $(0,+\infty)$ for all $t \geq 0$ a.s., i.e. it does not explode in finite time and the point 0 is not attainable for the solution a.s.

We prove it in two steps. First, we prove this statement for the solution $X^{\widetilde{u}}=\widetilde{X}$ to 6.1 computed for an arbitrary but fixed constant control $\widetilde{u} \in U$. To proceed define for every $n \in \mathbb{N}$ and $\delta>0$ two stopping times

$$
\begin{aligned}
& \tau_{n}=\inf \left\{t>0:\left|\widetilde{X}_{t}\right| \geq n\right\}, \\
& \tau_{\delta}=\inf \left\{t>0:\left|\widetilde{X}_{t}\right| \leq \delta\right\} .
\end{aligned}
$$

Note that by a.s. continuity of $\widetilde{X}, \tau_{n}$ is a nondecreasing sequence of times almost surely and $\tau_{\delta}$ is a nondecreasing with $\delta$ decreasing to 0 . Therefore there exist stopping times $\varepsilon$ (explosion time) and $\kappa$ (hitting time of 0 ) so that

$$
\begin{array}{ll}
\tau_{n} \nearrow \varepsilon, & n \rightarrow+\infty, \text { a.s. } \\
\tau_{\delta} \nearrow \kappa, & \delta \rightarrow 0_{+}, \text {a.s. }
\end{array}
$$

Further, the infinitesimal generator of the Markov semigroup corresponding to $\widetilde{X}$ is given by the formula

$$
\mathcal{L} f(x)=x F(x, \widetilde{u}) \frac{d}{d x} f(x)+\frac{1}{2} \sigma^{2}(x) \frac{d^{2}}{d x^{2}} f(x),
$$


for $f \in \mathcal{C}^{2}\left(\mathbb{R}_{+}\right)$. Using the Lyapunov function $V(x)=1+\frac{1}{x}+x^{2}$ and assumptions on $\sigma(\cdot)$ and $F(\cdot, \cdot)$ one gets

$$
\mathcal{L} V(x)=x F(x, \widetilde{u})\left(-\frac{1}{x^{2}}+2 x\right)+\frac{1}{2} \sigma^{2}(x)\left(\frac{2}{x^{3}}+2\right) \leq K\left(1+\frac{1}{x}+x^{2}\right)=K V(x),
$$

where $K=\max \left\{L^{2}+C, L^{2}+2 \mu_{1}\right\}$ for $x \in(0, r)$ and $K=\max \left\{L^{2}, 2 C+L^{2}\right\}$ for $x>R$.

Further, $V(x)$ is radially unbounded, i.e.

$$
\begin{aligned}
q_{R} & =\inf _{x \geq R} V(x)=1+R^{2} \rightarrow+\infty \quad \text { as } \quad R \rightarrow+\infty, \\
q_{\delta} & =\inf _{x \in(0, \delta]} V(x)=1+\frac{1}{\delta}+\delta^{2} \rightarrow+\infty \quad \text { as } \quad \delta \rightarrow 0_{+} .
\end{aligned}
$$

Now applying Itô formula to $V\left(\widetilde{X}_{t \wedge \tau_{n} \wedge \tau_{\delta}}\right)$ and taking $\mathbf{E}(\cdot)$ one arrives at

$$
\begin{aligned}
e^{K t} V\left(x_{0}\right) & \geq \mathbf{E}\left[V\left(\widetilde{X}_{t \wedge \tau_{n} \wedge \tau_{\delta}}\right)\right]=\mathbf{E}\left[\mathbf{1}_{\left\{\tau_{n} \wedge \tau_{\delta} \leq t\right\}} V\left(\tilde{X}_{\tau_{n} \wedge \tau_{\delta}}\right)\right]+\mathbf{E}[\underbrace{\mathbf{1}_{\left\{\tau_{n} \wedge \tau_{\delta} \geq t\right\}} V\left(\tilde{X}_{t}\right)}_{\geq 0}] \\
& \geq \mathbf{E}[\underbrace{\mathbf{1}_{\left\{\tau_{n} \wedge \tau_{\delta} \leq t\right\}} \mathbf{1}_{\left\{\tau_{\delta} \leq \tau_{n}\right\}}}_{=\mathbf{1}_{\left\{\tau_{\delta} \leq t\right\}}} \underbrace{V\left(\widetilde{X}_{\tau_{\delta}}\right)}_{\geq q_{\delta}}]+\mathbf{E}[\underbrace{\mathbf{1}_{\left\{\tau_{n} \wedge \tau_{\delta} \leq t\right\}} \mathbf{1}_{\left\{\tau_{\delta} \geq \tau_{n}\right\}}}_{=\mathbf{1}_{\left\{\tau_{n} \leq t\right\}}} \underbrace{V\left(\widetilde{X}_{\tau_{n}}\right)}_{\geq q_{n}}] \\
& \geq \mathbb{P}\left(\tau_{\delta} \leq t\right) q_{\delta}+\mathbb{P}\left(\tau_{n} \leq t\right)
\end{aligned}
$$

Therefore, employing (6.8) we have for each $t \geq 0$ fixed

$$
\begin{aligned}
& \mathbb{P}\left(\tau_{n} \leq t\right) \leq \frac{e^{K t} V\left(x_{0}\right)}{q_{n}} \underset{n \rightarrow+\infty}{\longrightarrow} 0, \\
& \mathbb{P}\left(\tau_{\delta} \leq t\right) \leq \frac{e^{K t} V\left(x_{0}\right)}{q_{\delta}} \underset{\delta \rightarrow 0_{+}}{\longrightarrow} 0 .
\end{aligned}
$$

Taking into account 6.7 and 6.10) we arrive at

$$
\max \{\mathbb{P}(\varepsilon \leq t), \mathbb{P}(\kappa \leq t)\}=0, \quad \forall t \geq 0 .
$$

Sending $t \rightarrow+\infty$, we finally obtain

$$
\max \{\mathbb{P}(\varepsilon<+\infty), \mathbb{P}(\kappa<+\infty)\}=0,
$$

which means that with probability 1 the solution $\widetilde{X}$ to equation 6.1 computed for some fixed constant control $\widetilde{u} \in U$ and for $x_{0}>0$ does not explode and does not attain 0 in finite time.

In the second step we show that for any admissible control $u(\cdot) \in \mathcal{U}_{a d}$, the solution $X=X^{u(\cdot)}$ to equation 6.1) satisfies

$$
X_{t}^{u_{\text {lower }}} \leq X_{t}^{u(\cdot)} \leq X_{t}^{u_{\text {upper }}}, \forall t \geq 0,
$$

for the common initial condition $x_{0}>0$.

The result of the previous step holds in particular for $X^{u_{\text {lower }}}$ and $X^{u_{\text {upper }}}$. For proving the unattainability of 0 , a comparison theorem will be the key tool. We employ Theorem 1.1 and Remark 1.1 in Ikeda, Watanabe, [13] for special choice of functions

$$
\begin{aligned}
& \beta_{1}(t, \omega)=b_{1}\left(t, X_{t}^{u_{\text {lower }}}\right)=b_{2}\left(t, X_{t}^{u_{\text {lower }}}\right)=X_{t}^{u_{\text {lower }}} F\left(X_{t}^{u_{\text {lower }}}, u_{\text {lower }}\right), \\
& \beta_{2}(t, \omega)=X_{t}^{u(\cdot)} F\left(X_{t}^{u(\cdot)}, u(t)\right) .
\end{aligned}
$$

Using (6.3) we know that 


$$
X^{u(\cdot)}\left(t \wedge \tau_{\delta}^{\prime}\right) F\left(X^{u(\cdot)}\left(t \wedge \tau_{\delta}^{\prime}\right), u_{\text {lower }}\right) \leq X^{u(\cdot)}\left(t \wedge \tau_{\delta}^{\prime}\right) F\left(X^{u(\cdot)}\left(t \wedge \tau_{\delta}^{\prime}\right), u\left(t \wedge \tau_{\delta}^{\prime}\right),\right)
$$

for all $t \geq 0, x_{0}>0$ and $\tau_{\delta}^{\prime}=\inf \left\{t>0:\left|X_{t}^{u(\cdot)}\right| \leq \delta\right\}, \delta>0$. Applying Theorem 1.1 and Remark 1.1 in [13] one gets

$$
0<X^{u_{\text {lower }}}(s) \leq X^{u(\cdot)}(s), \forall s \in\left[0, \tau_{\delta}^{\prime}\right), \forall \delta>0 .
$$

From the construction of stopping times $\tau_{\delta}^{\prime}, \tau_{\delta}$ (the latter now defined in terms of the process $X^{u_{\text {lower }}}$ ), by 6.15 and from the first step of the proof it follows that

$$
0<\tau_{\delta} \leq \tau_{\delta}^{\prime}
$$

for all $\delta>0$. Since $\tau_{\delta} \rightarrow+\infty$ almost surely as $\delta \rightarrow 0_{+}$we conclude that $X^{u(\cdot)}$ does not attain 0 for all $t>0$ almost surely. Nonexplosion of $X^{u(\cdot)}$ can be proved similarly.

By Remark 3 we conclude that Theorem 4 (or Theorem 5 can be applied for the above DCP.

6.1. The controlled logistic equation. In the rest of the section we derive the form of the associated FBSDE and the Hamiltonian for a special choice of $F(\cdot, \cdot)$, namely for $x F(x, u)=a x(1-b x)+\gamma u$, for $a, b>0, u \in\left[u_{1}, u_{2}\right], u_{1}>0, \gamma \in \mathbb{R}$, i.e. the additive control is one dimensional process. The diffusion term is given by $\sigma(x)=\sigma x$ for some $\sigma>0$. The one dimensional controlled diffusion therefore evolves according to the SDE

$$
\begin{aligned}
d X_{t} & =a X_{t}\left(1-b X_{t}\right) d t+\gamma u_{t} d t+\sigma X_{t} d W_{t}, \quad \forall t \geq 0, \\
X_{0} & =x_{0}>0 .
\end{aligned}
$$

The functional to be minimized over all admissible controls $u(\cdot)$ is

$$
J(u(\cdot))=\mathbf{E} \int_{0}^{+\infty} e^{-\beta t}\left(c X_{t}^{2}+h u_{t}^{2}\right) d t,
$$

where $c, h>0$ and $\beta>0$ is a discount factor. Again, we will maximize $-J(u(\cdot))$ and we see that the Hamiltonian is of the form

$$
\mathcal{H}(x, u, y, z)=a\left(x-b x^{2}\right) y+\gamma u y+\sigma x z-c x^{2}-h u^{2}-\beta x y,
$$

which is a concave function in $(x, u)$. The driver of the backward adjoint equation is obtained as the derivative of $\mathcal{H}$ w.r.t. $x$, i.e.

Therefore, the associated BSDE reads

$$
\frac{\partial}{\partial x} \mathcal{H}(x, u, y, z)=(a-\beta) y-2 a b x y+\sigma z-2 c x .
$$

$$
-d Y_{t}=\left[(a-\beta) Y_{t}-2 a b X_{t} Y_{t}+\sigma Z_{t}-2 c X_{t}\right] d t-Z_{t} d W_{t}, \quad \forall t \geq 0 .
$$

To find the maximal point of $\mathcal{H}$ w.r.t. $u$ we lay

$$
\frac{\partial}{\partial u} \mathcal{H}(x, u, y, z)=\gamma y-2 h u=0,
$$

to obtain the argmax to the quadratic function in $u$ given by $\mathcal{H}$, i.e.

$$
u_{\max }=\frac{\gamma}{2 h} y .
$$

Finally, the optimal control is obtained by studying the mutual position of $u_{\max }$ and the interval $\left[u_{1}, u_{2}\right]$ which leads to the optimal control

$$
\widehat{u}_{t}=\widetilde{u}\left(Y_{t}\right)
$$

where 


$$
\widetilde{u}(y)=\left\{\begin{array}{l}
u_{1}, y \leq \frac{2 h}{\gamma} u_{1}, \\
\frac{\gamma}{2 h} y, y \in\left[\frac{2 h}{\gamma} u_{1}, \frac{2 h}{\gamma} u_{2}\right], \\
u_{2}, y \geq \frac{2 h}{\gamma} u_{2} .
\end{array}\right.
$$

It can be easily seen that $\widehat{u}(\cdot)$ is bounded by $u_{2}$, continuous and nondecreasing. The last thing which is not obvious is that $\widehat{u}_{t}$ is indeed an admissible control, i.e. that FBSDE 6.17, 6.20) admits a unique solution with $\widehat{u}_{t}$ plugged. When this is shown then according to Theorem 4 (or Theorem 5) $\widehat{u}_{t}$ is optimal.

The corresponding FBSDE in the present case reads

$$
\begin{aligned}
d X_{t} & =a X_{t}\left(1-b X_{t}\right) d t+\gamma \widetilde{u}\left(Y_{t}\right) d t+\sigma X_{t} d W_{t}, \\
-d Y_{t} & =\left[(a-\beta) Y_{t}-2 a b X_{t} Y_{t}+\sigma Z_{t}-2 c X_{t}\right] d t-Z_{t} d W_{t}, \\
X_{0} & =x_{0}>0 .
\end{aligned}
$$

FBSDE with the non-Lipschitz term $-2 a b x y$ in the driver of the backward part are not covered (up to authors' knowledge) with the existing theory.

We start with the local uniqueness theorem proved for the solution in the space $\mathbf{L}_{\mathcal{F}, \text { loc }}^{2}\left(\mathbb{R}_{+} ; \mathbb{R}^{3}\right)$ which is sufficient for finite time horizon problem.

Lemma 3 (Local uniqueness). Let $\left(X_{t}^{i}, Y_{t}^{i}, Z_{t}^{i}\right)_{t \in\left[t_{0}, t_{0}+\delta\right]}, i=1,2$ be two solution to FBSDE 6.24) for some $\delta>0, t_{0} \geq 0$ such that $X_{t_{0}}^{1}=X_{t_{0}}^{2}$ and $Y_{t_{0}+\delta}^{1}=Y_{t_{0}+\delta}^{2}$. Then

$$
\left(X_{t}^{1}, Y_{t}^{1}, Z_{t}^{1}\right)=\left(X_{t}^{2}, Y_{t}^{2}, Z_{t}^{2}\right), \forall t \in\left[t_{0}, t_{0}+\delta\right], \mathbb{P}-a . s .
$$

Proof: Denoting $\widehat{X}_{t}=X_{t}^{2}-X_{t}^{1}, \widehat{Y}_{t}=Y_{t}^{2}-Y_{t}^{1}, \widehat{Z}_{t}=Z_{t}^{2}-Z_{t}^{1}, \forall t \in\left[t_{0}, t_{0}+\delta\right]$, applying Itô formula and using standard estimates one obtains

$$
\begin{gathered}
\sup _{t \in\left[t_{0}, t_{0}+\delta\right]} \mathbf{E}\left|\widehat{X}_{t}\right|^{2} \leq C\left[\mathbf{E}\left|\widehat{X}_{t_{0}}\right|^{2}+\mathbf{E}\left|\widehat{Y}_{t_{0}+\delta}\right|^{2}\right] \exp \left(K \delta+K \mathbf{E} \int_{t_{0}}^{t_{0}+\delta}\left|Y_{s}^{1}\right|^{2} d s\right) \\
\sup _{t \in\left[t_{0}, t_{0}+\delta\right]} \mathbf{E}\left|\widehat{Y}_{t}\right|^{2}+\left(1-\frac{\sigma}{\varepsilon_{1}}\right) \mathbf{E} \int_{t_{0}}^{t_{0}+\delta}\left|\widehat{Z}_{s}\right|^{2} d s \leq C\left[\mathbf{E}\left|\widehat{Y}_{t_{0}+\delta}\right|^{2}+\mathbf{E} \int_{t_{0}}^{t_{0}+\delta}\left|\widehat{X}_{s}\right|^{2} d s\right] \\
\quad \times \exp \left(K \delta+K \mathbf{E} \int_{t_{0}}^{t_{0}+\delta}\left|\widehat{X}_{t}\right|^{2} d t\right)
\end{gathered}
$$

where $C, K>0$ are some constants and $\varepsilon_{1}>0$ is sufficiently small.

In a similar way one obtains the estimates with $\sup (\cdot)$ inside the expectation

$$
\begin{gathered}
\left(1-\varepsilon_{2} \frac{C_{1}}{2}\right) \mathbf{E}\left[\sup _{t \in\left[t_{0}, t_{0}+\delta\right]}\left|\widehat{X}_{t}\right|^{2}\right] \leq C\left[\mathbf{E}\left|\widehat{X}_{t_{0}}\right|^{2}+\mathbf{E}\left|\widehat{Y}_{t_{0}+\delta}\right|^{2}\right] \exp \left(K \delta+K \mathbf{E} \int_{t_{0}}^{t_{0}+\delta}\left|Y_{s}^{1}\right|^{2} d s\right) \\
\left(1-2 C_{1} \varepsilon_{3}\right) \mathbf{E}\left[\sup _{t \in\left[t_{0}, t_{0}+\delta\right]}\left|\widehat{Y}_{t}\right|^{2}\right] \\
+C \mathbf{E} \int_{t_{0}}^{t_{0}+\delta}\left|Y_{s}^{1}\right|^{2} \cdot\left|\widehat{X}_{s}\right|^{2} d s
\end{gathered}
$$

where again $C, K>0$ are some constants, $C_{1}>0$ is the constant from Burkholder-Davis-Gundy inequality and $\varepsilon_{1}, \varepsilon_{2}, \varepsilon_{3}>0$ are some sufficiently small numbers.

Since $\widehat{X}_{t_{0}}=\widehat{Y}_{t_{0}+\delta}=0$ uniqueness of the process $X$ follows from 6.26. Uniqueness of the processes $Y, Z$ then follows from 6.27). 
To sketch the construction of the solution we first introduce for each $n \in \mathbb{N}$ the approximated equation

$$
\begin{aligned}
d X_{t}^{n} & =a X_{t}^{n}\left(1-b X_{t}^{n}\right) d t+\gamma \widetilde{u}\left(Y_{t}^{n}\right) d t+\sigma X_{t}^{n} d W_{t}, \\
-d Y_{t}^{n} & =\left[(a-\beta) Y_{t}^{n}-2 a b\left(X_{t}^{n} \wedge n\right) Y_{t}^{n}+\sigma Z_{t}^{n}-2 c\left(X_{t}^{n} \wedge n\right)\right] d t-Z_{t}^{n} d W_{t}, \\
X_{0}^{n} & =x_{0}>0 .
\end{aligned}
$$

The second equation in the system (6.28) has Lipschitzian r.h.s. and it is well known (Yin, [26]) that it admits a unique solution $\left(X_{t}^{n}, Y_{t}^{n}, Z_{t}^{n}\right)_{t>0}$ in $\mathbf{L}_{\mathcal{F}}^{2,-\beta}\left(\mathbb{R}_{+} ; \mathbb{R}^{3}\right)$ for each $n \in \mathbb{N}$ and for some exponential weight $\beta>0$ specified later. We also note that the approximate drivers $h_{n}(x, y, z)=$ $(a-\beta) y-2 a b(x \wedge n) y+\sigma z-2 c(x \wedge n)$ converge pointwise to the original driver $h(x, y, z)=$ $(a-\beta) y-2 a b x y+\sigma z-2 c x$ as $n \rightarrow+\infty$ and that the solution process $X$ inherits all the properties (nonexplosion etc.) proved above using the Lyapunov function.

Using standard techniques it is not difficult to derive the following uniform estimates (in $n$ ) of the process $X$ for $\beta>\sigma^{2}+2 a+\frac{C_{1} \sigma}{\varepsilon_{3}}+\max \left\{\gamma u_{2}, \frac{\gamma \varepsilon}{2}\right\}$

$$
\begin{aligned}
& \sup _{t \geq 0} \mathbf{E}\left[e^{-\beta t}\left|X_{t}^{n}\right|^{2}\right]+\left(1-C_{1} \sigma \varepsilon_{3}\right) \mathbf{E}\left[\sup _{t \geq 0} e^{-\beta t}\left|X_{t}^{n}\right|^{2}\right] \leq x_{0}^{2}+\frac{\gamma u_{2}}{\beta}<+\infty, \\
& \sup _{t \geq 0} \mathbf{E}\left[e^{-\beta t}\left|\widehat{X}_{t}\right|^{2}\right]+\left(1-C_{1} \sigma \varepsilon_{3}\right) \mathbf{E}\left[\sup _{t \geq 0} e^{-\beta t}\left|\widehat{X}_{t}\right|^{2}\right] \leq \frac{\gamma^{3}}{h^{2} \varepsilon} \mathbf{E} \int_{0}^{+\infty} e^{-\beta s}\left|\widehat{Y}_{s}\right|^{2} d s,
\end{aligned}
$$

where $\varepsilon, \varepsilon_{3}>0$ are some sufficiently small numbers and $\widehat{X}=X^{m}-X^{n}, \widehat{Y}=Y^{m}-Y^{n}$ for each $m, n \in \mathbb{N}$.

Now, let $n, N \in \mathbb{N}$ be arbitrary and $t \geq 0$. Using previous estimates and Chebyshev inequality one can examine the boundedness of the process $X$ in probability (uniformly in $t$ on any finite time interval $[0, T]$ for each $T>0$ fixed) in the following meaning

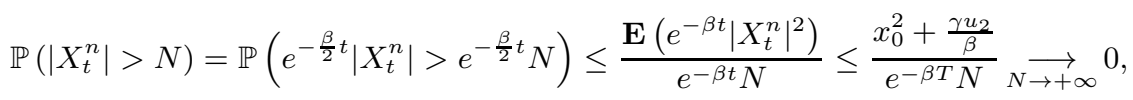

which will be a corner stone for the construction of the solution on finite time horizon. Therefore we consider the finite time horizon approximated equation

$$
\begin{aligned}
d X_{t}^{n} & =a X_{t}^{n}\left(1-b X_{t}^{n}\right) d t+\gamma \widetilde{u}\left(Y_{t}^{n}\right) d t+\sigma X_{t}^{n} d W_{t}, t \in(0, T] \\
-d Y_{t}^{n} & =\left[(a-\beta) Y_{t}^{n}-2 a b\left(X_{t}^{n} \wedge n\right) Y_{t}^{n}+\sigma Z_{t}^{n}-2 c\left(X_{t}^{n} \wedge n\right)\right] d t-Z_{t}^{n} d W_{t}, t \in[0, T) \\
X_{0}^{n} & =x_{0}>0, \\
Y_{T}^{n} & =0 .
\end{aligned}
$$

Employing classical results on BSDE we know that there is a unique the solution to (6.32) in $\mathbf{L}_{\mathcal{F}}^{2,-\beta}\left([0, T] ; \mathbb{R}^{3}\right)$ for each $T>0$ fixed and for each $n \in \mathbb{N}$.

Further, let $\Gamma_{N}$ be a cylindrical subset of $\mathbb{R}^{3}$ of the form

$$
\Gamma_{N}=B(0, N) \times \mathbb{R} \times \mathbb{R}
$$

where $B(0, N)$ is a ball of radius $N \in \mathbb{N}$ with center in origin. Now we are ready to prove the consistency theorem.

Theorem 6. [Consistency] Let $T>0$ be arbitrary but fixed, $N \in \mathbb{N}$ be some (large enough) index and $m, p \in \mathbb{N}, m, p>N$. Let $\beta>\sigma^{2}+2 a+\frac{C_{1} \sigma}{\varepsilon_{3}}+\max \left\{\gamma u_{2}, \frac{\gamma \varepsilon}{2}\right\}$ and let $\left(X_{t}^{m}, Y_{t}^{m}, Z_{t}^{m}\right)_{t \in[0, T]}$ and $\left(X_{t}^{p}, Y_{t}^{p}, Z_{t}^{p}\right)_{t \in[0, T]}$ respectively be two solutions to FBSDE [6.32] in $\mathbf{L}_{\mathcal{F}}^{2,-\beta}\left([0, T] ; \mathbb{R}^{3}\right)$ computed for $n=m, n=p$ respectively. Then

$$
\left(X_{t}^{m}, Y_{t}^{m}, Z_{t}^{m}\right)(\omega)=\left(X_{t}^{p}, Y_{t}^{p}, Z_{t}^{p}\right)(\omega), \forall t \in[0, T], \forall \omega \in \Omega_{\Gamma_{N}},
$$


where $\Omega_{\Gamma_{N}}=\left\{\omega \in \Omega:\left(X_{t}^{m}, Y_{t}^{m}, Z_{t}^{m}\right)(\omega) \in \Gamma_{N},\left(X_{t}^{p}, Y_{t}^{p}, Z_{t}^{p}\right)(\omega) \in \Gamma_{N}, \forall t \in[0, T]\right\}$.

In other words, all the trajectories which stay in the cylinder $\Gamma_{N}$ coincide.

Proof: Let $\omega \in \Omega_{\Gamma_{N}}$ and denote $V_{t}^{n}=\left(X_{t}^{n}, Y_{t}^{n}, Z_{t}^{n}\right)$ for all $n \in \mathbb{N}$. Then for $\left(V_{t}^{p}\right)(\omega)$ and $\left(V_{t}^{m}\right)(\omega), m>p$, we obtain

$$
\begin{gathered}
Y_{t}^{p}(\omega)=\int_{t}^{T} h_{p}\left(V_{s}^{p}(\omega)\right) \underbrace{\mathbf{1}_{\Gamma_{p}}\left(V_{s}^{p}(\omega)\right)}_{=1} d s+\int_{t}^{T} h_{p}\left(V_{s}^{p}(\omega)\right) \underbrace{\mathbf{1}_{\Gamma_{p}^{c}}\left(V_{s}^{p}(\omega)\right)}_{=0} d s-\left(\int_{t}^{T} Z_{s}^{p} d W_{s}\right)(\omega), \\
Y_{t}^{m}(\omega)=\int_{t}^{T} h_{m}\left(V_{s}^{m}(\omega)\right) \underbrace{\mathbf{1}_{\Gamma_{m}}\left(V_{s}^{m}(\omega)\right)}_{=1} d s+\int_{t}^{T} h_{m}\left(V_{s}^{m}(\omega)\right) \underbrace{\mathbf{1}_{\Gamma_{m}^{c}}\left(V_{s}^{m}(\omega)\right)}_{=0} d s \\
-\left(\int_{t}^{T} Z_{s}^{m} d W_{s}\right)(\omega) .
\end{gathered}
$$

We also notice that

$$
\begin{aligned}
h_{p}(x, y, z) \mathbf{1}_{\Gamma_{p}}(x, y, z) & =h_{m}(x, y, z) \mathbf{1}_{\Gamma_{p}}(x, y, z)=h(x, y, z) \mathbf{1}_{\Gamma_{p}}(x, y, z) \text { and } \\
h_{p}(x, y, z) \mathbf{1}_{\Gamma_{N}}(x, y, z) & =h_{m}(x, y, z) \mathbf{1}_{\Gamma_{N}}(x, y, z)=h_{N}(x, y, z) \mathbf{1}_{\Gamma_{N}}(x, y, z) .
\end{aligned}
$$

Since $\Gamma_{N} \subset \Gamma_{p} \subset \Gamma_{m}$ for $m>p>N,\left(V_{t}^{p}\right)(\omega)$ also satisfies the same integral relation 6.35) and the forward equation of the system, i.e. $\left(V_{t}^{p}\right)(\omega)$ solves (6.35) as well, and by the local uniqueness (Lemma3 with $n=m$ ) it follows that $\left(V_{t}^{p}\right)(\omega)=\left(V_{t}^{m}\right)(\omega), \forall t \in[0, T]$.

Further, we can prove along similar lines that $\left(V_{t}^{p}\right)(\omega),\left(V_{t}^{m}\right)(\omega)$ solve 6.32 for $n=N$. Putting both arguments together we see that all the solution trajectories that stay in some cylinder (for some index) solve also all the equation with larger index and all such trajectories coincide.

Proposition 1 (Construction of the solution). Under the assumptions of Theorem 6 there exists a unique solution $\left(X_{t}, Y_{t}, Z_{t}\right)_{[0, T]} \in \mathbf{L}_{\mathcal{F}}^{2,-\beta}\left(\mathbb{R}_{+} ; \mathbb{R}^{3}\right)$ of the finite horizon FBSDE

$$
\begin{aligned}
d X_{t} & =a X_{t}\left(1-b X_{t}\right) d t+\gamma \widetilde{u}\left(Y_{t}\right) d t+\sigma X_{t} d W_{t}, \\
-d Y_{t} & =\left[(a-\beta) Y_{t}-2 a b X_{t} Y_{t}+\sigma Z_{t}-2 c X_{t}\right] d t-Z_{t} d W_{t}, \\
X_{0} & =x_{0}>0, \\
Y_{T} & =0 .
\end{aligned}
$$

Proof: Keeping the notation from the previous proof notice that

$$
\Omega_{\Gamma_{N}}=\Omega_{p, N} \cap \Omega_{m, N},
$$

where $\Omega_{n, N}=\left\{\omega \in \Omega:\left(X_{t}^{n}, Y_{t}^{n}, Z_{t}^{n}\right)(\omega) \in \Gamma_{N}, \forall t \in[0, T]\right\}$, for $n \in \mathbb{N}$ and due to estimate 6.31) we have that $\mathbb{P}\left(\Omega_{p, N}\right) \underset{N \rightarrow+\infty}{\longrightarrow} 1$ uniformly in $p$. Thus we put for any fixed $p>N$

$$
\widetilde{\Omega}=\bigcup_{N \in \mathbb{N}} \Omega_{p, N}, \text { with } \mathbb{P}(\widetilde{\Omega})=1
$$

The unique limit solution process to FBSDE

$$
\begin{aligned}
d X_{t} & =a X_{t}\left(1-b X_{t}\right) d t+\gamma \widetilde{u}\left(Y_{t}\right) d t+\sigma X_{t} d W_{t}, t \in(0, T] \\
-d Y_{t} & =\left[(a-\beta) Y_{t}-2 a b X_{t} Y_{t}+\sigma Z_{t}-2 c X_{t}\right] d t-Z_{t} d W_{t}, t \in[0, T) \\
X_{0} & =x_{0}>0, \\
Y_{T} & =0 .
\end{aligned}
$$

is obtained for all $t \in[0, T]$ as 


$$
\left(X_{t}, Y_{t}, Z_{t}\right)(\omega)= \begin{cases}\left(X_{t}^{N}, Y_{t}^{N}, Z_{t}^{N}\right)(\omega), & \text { for } \omega \in \Omega_{p, N}, \\ \left(X_{t}^{N+1}, Y_{t}^{N+1}, Z_{t}^{N+1}\right)(\omega), & \text { for } \omega \in \Omega_{p, N+1} \backslash \Omega_{p, N},\end{cases}
$$

for each $N \in \mathbb{N}$. We stress that this construction is independent of the choice of $p$ due to the consistency theorem.

Construction of the solution to the original FBSDE 6.24 now follows the lines of analogous proof in Pardoux [20] with the process $\left(X_{t}, Y_{t}, Z_{t}\right)$ from Proposition 1 as its finite time horizon approximation. That is, the solution to $(6.24)$ is obtained as a limit of solutions $\left(X_{t}^{n}, Y_{t}^{n}, Z_{t}^{n}\right)_{t \geq 0}$ where

$$
\begin{aligned}
d X_{t}^{n} & =a X_{t}^{n}\left(1-b X_{t}^{n}\right) d t+\gamma \widetilde{u}\left(Y_{t}^{n}\right) d t+\sigma X_{t}^{n} d W_{t}, t \in(0, n] \\
-d Y_{t}^{n} & =\left[(a-\beta) Y_{t}^{n}-2 a b X_{t}^{n} Y_{t}^{n}+\sigma Z_{t}^{n}-2 c X_{t}^{n}\right] d t-Z_{t}^{n} d W_{t}, t \in[0, n) \\
X_{0}^{n} & =x_{0}>0, \\
X_{t}^{n} & =X_{n}^{n}, t \geq n, \\
Y_{t}^{n} & =0, t \geq n \\
Z_{t}^{n} & =0, t \geq n .
\end{aligned}
$$

\section{REFERENCE}

[1] Agram N., Haadem S., Øksendal B. and Proske F. A maximum principle for infinite horizon delay equations. In preparation.

[2] Al-Hussein A. Maximum Principle for Controlled Stochastic Evolution Equations. Int. Journal of Math. Analysis. 4, 1447-1464, 2010

[3] Bahlali S. and Mezerdi B. A General stochastic maximum principle for singular control problems. Electronic Journal of Probability 10, 988-1004, 2005

[4] Bensoussan A. Maximum principle and dynamic programming approaches of the optimal control of partially observed diffusions. Stochastics. 9, 169-222, 1983.

[5] Bismut J.-M. Conjugate convex functions in optimal stochastic control. J. Math. Anal. Appl. 44, 384-404, 1973.

[6] Borkar V. S. Controlled diffusion processes. Probability Surveys. 2, 213-244, 2005.

[7] Cadenillas A. and Haussmann U. G. The stochastic maximum principle for a singular control problem. Stochastics Rep. 49, 211-237, 1994.

[8] Chikodza E. COMBINED SINGULAR AND IMPULSE CONTROL FOR JUMP DIFFUSIONS. SAMSA Journal of Pure and Applied Mathematics. 3, 29-57, 2008

[9] Dufour, F. and Miller, B. Maximum principle for singular stochastic control problems. SIAM J. Control Optim. 45, 668-698, 2006

[10] Friedman A. Stochastic Differential Equations and Applications: Volume 1. Academic Press, 1975

[11] Fuhrman M, Hu Y. and Tessitore G. Stochastic maximum principle for optimal control of SPDEs. Comptes Rendus Mathematique. 350, 683-688, 2012

[12] Haadem S., Proske F. and Øksendal B. Maximum principles for jump diffusion processes with infinite horizon. http://arxiv.org/pdf/1206.1719.pdf

[13] Ikeda, N. and Watanabe. S. A COMPARISON THEOREM FOR SOLUTIONS OF STOCHASTIC DIFFERENTIAL EQUATIONS AND ITS APPLICATIONS. Osaka J. Math. 14, 619-633, 1977.

[14] Kushner H. J. Necessary conditions for continuous parameter stochastic optimization problems. SIAM J. Control. 10, 550-565, 1972.

[15] Øksendal B. Stochastic Differential Equations. An Introduction with Applications, 4th edition. Berlin, Springer-Verlag 1995., ISBN 3-540-60243-7 (Universitext)

[16] Øksendal B. and Sulem A. Singular stochastic control and optimal stopping with partial information of Itô-Lévy processes. SIAM J. Control Optim. 50, 2254-2287, 2012

[17] Øksendal B., Sulem A. and Framstad N. C. A sufficient stochastic maximum principle for optimal control of jump diffusions and applications to finance. J. Optimization Theory and Applications, 121, 77-98, 2004. Errata: J. Optimization Theory and Applications 124, 511-512, 2005.

[18] Øksendal B., Sulem A. and Zhang T. OPTIMAL CONTROL OF STOCHASTIC DELAY EQUATIONS AND TIME-ADVANCED BACKWARD STOCHASTIC DIFFERENTIAL EQUATIONS. Int. Advances in Applied Probability. 43, 572-596, 2011

[19] Pardoux E. BSDEs weak convergence and homogenizations of semilinear PDEs. Nonlinear Analysis Differential Equations and Control. Clark, F.H., Stern, R.J. (Eds.), Kluwer Academic, Dordrecht, 503-549, 1999.

[20] Pardoux E., R. W. R. Darling Backwards SDE with Random Terminal Time and Applications to Semilinear Elliptic PDE. The Annals of Probability. Vol. 25, No. 3, 1135-1159, 1997. 
[21] Pardoux E., Peng S. G. Adapted solution of a backward stochastic differential equation. Systems \& Control Letters. 14, 55-61, 1990.

[22] Peng S. G. A general stochastic maximum principle for optimal control problems. SIAM J. Control Optim. 28, 966-979, 1990

[23] Peng S. G., Shi Y. Infinite horizon forward-backward stochastic differential equations. Stochastic Process. Appl. 85, 75-92, 2000.

[24] Pham, H. Continuous-time stochastic control and optimization with financial applications. Stochastic Modelling and Applied Probability 61, Springer-Verlag, Berlin, 2009.

[25] Tang S. J. and Li X. J. Necessary conditions for optimal control of stochastic systems with random jumps. SIAM J. Control Optim. 32, 1447-1475, 1994.

[26] Yin J. On solutions of a class of infinite horizon FBSDE's. Statistics and Probability Letters. 78, 2412-2419, 2008.

[27] Wu Z. Forward-Backward Stochastic Differential Equations with Stopping Time. ACTA MATHEMATICA SCIENTIA. 24, 91-99, 2004.

[28] Wu Z. and Zhang F. Maximum Principle for Stochastic Recursive Optimal Control Problems Involving Impulse Controls. Abstract \& Applied Analysis. 32, 1-16, 2012

[29] Zhou X.Y. STOCHASTIC NEAR-OPTIMAL CONTROLS: NECESSARY AND SUFFICIENT CONDITIONS FOR NEAR-OPTIMALITY. SIAM J. Control Optim. 36, 929-947, 1998 